\title{
Buenos Aires, entre Eros y Tánatos. La prostitución como amenaza disgénica (1930-1955)
}

\author{
Marisa A. Miranda (*) \\ $\left({ }^{*}\right) \quad$ IIB-INTECH/CONICET-UNSAM. Chascomús, Buenos Aires. \\ mmiranda2804@gmail.com
}

Dynamis

[0211-9536] 2012; $32(1): 93-113$

Fecha de recepción: 9 de marzo de 2011

Fecha de aceptación: 5 de septiembre de 2011

SUMARIO: 1.-Introducción: la prostitución femenina como amenaza disgénica. 2.-Mal venéreo y defensa social. 3.-La eugenesia y el anclaje biopolítico del abolicionismo. 4.-Reflexiones finales.

RESUMEN: El presente trabajo explora el discurso médico-legal construido en Buenos Aires durante las primeras décadas del siglo XX en torno a la prostitución femenina. A partir de los significativos paralelismos entablados por entonces entre cuerpos prostituidos, enfermedad y delito, se ahonda sobre los diversos recursos ideológicos que sustentaron las, a veces disímiles, políticas articuladas en pos del control del comercio sexual, haciendo particular hincapié en el análisis de la estigmatización sociocultural y, en consecuencia, también jurídica, de las mujeres "de mala vida» de la cual, claro está, quedaban empero exentos los hombres que utilizaban sus servicios. En este orden, cabe recordar que la emblemática capital de Argentina se había constituido ya hacia fines del siglo XIX en un polo de trata de blancas con reconocimiento internacional. Sobre esta circunstancia, evidentemente cierta, se estructuraron planteos xenófobos vinculados a la participación judía en las organizaciones de proxenetas que resultaron funcionales al fortalecimiento de actitudes hostiles hacia lo «diverso»; y donde la medicina y el derecho aportaron insumos fundamentales para un enfoque hipersimplificado de la compleja problemática de la servidumbre sexual.

PALABRAS CLAVE: Buenos Aires, Argentina, prostitución, eugenesia, biopolítica.

KEY WORDS: Buenos Aires, Argentina, prostitution, eugenics, biopolitics. 


\section{Introducción: la prostitución femenina como amenaza disgénica $\left(^{*}\right)$}

La exhumación de las apropiaciones biopolíticas del cuerpo en perspectiva histórica permite redefinir la mirada de lo singular subyacente a una «historia del cuerpo» y pensar, más allá de un sesgo homogeneizador, en «historias de los cuerpos» ${ }^{1}$. Desde esta perspectiva, una característica fundamental del siglo XX ha sido el tratamiento diferencial otorgado a los cuerpos jerarquizados, a los cuerpos «de arriba» y a los cuerpos «de abajo»; en definitiva, a los cuerpos serviles y a los cuerpos privilegiados, a los cuerpos «al servicio de» y a los cuerpos «que se sirven de». Adquiere visibilidad, entonces, la inmanencia de una gradación cultural presentada, ya desde finales del siglo XIX, como biológica.

En este marco, resulta entonces imprescindible el estudio de la prostitución. El cuerpo individual que define, identifica y sostiene a la entidad plural digna de preservación biopolítica - población, raza o grupo- fue predefinido como una categoría a tutelar mientras que, por oposición, el cuerpo individual que define, identifica y sostiene a la entidad plural digna de debilitamiento y desaparición biopolítica - población, raza o grupo-, fue sistemáticamente ignorado en relación a su protección en cuanto a sí mismo. Su función primordial comenzaba y terminaba en el afianzamiento de una jerarquía que, como tal, requería la presencia concreta de todos sus componentes, de los «de arriba»y de los «de abajo».

Sobre estas mismas ideas se encaró el control del negocio del sexo en el Buenos Aires de principios del siglo XX, cuya proliferación generaba un particular paisaje de la capital rioplatense, dotada por entonces de significativa presencia del componente inmigratorio masculino.

(*) Este trabajo forma parte de una investigación subsidiada por la ANPCyT (PICT-2007-01559) y el CONICET (PIP 114-200801-00065), de Argentina. Asimismo, se enmarca dentro del proyecto «Ciencia y creencia entre dos mundos. Evolucionismo, biopolítica y religión en España y Argentina», de referencia HAR2010-21333-C03-CO3, financiado por el Ministerio de Ciencia e Innovación (España).

1. Sobre esta cuestión resultan de ineludible cita los emblemáticos textos: Corbin, Alain; Courtine, Jean-Jacques; Vigarello, Georges, dirs. Historia del cuerpo. Vol. 3. Madrid: Taurus; 2006; y Porter, Roy. Historia del cuerpo. In: Burke, Peter, ed. Formas de hacer historia. Madrid: Alianza Universidad; 1994, p. 255-286. 
La historiografía que se ha venido ocupando de relevar estas cuestiones ha destacado diversos y trascendentales aspectos de esa realidad ${ }^{2}$. En efecto, desde el recuerdo de El Puente de los Suspiros, polémico periódico de efímera vida - se publicó en la capital argentina en 1878 y durante tan sólo tres meses- que aseguraba (¿falazmente?) denunciar a los burdeles, hasta el análisis de políticas locales asociadas al control de los prostíbulos, la revisión de aspectos socioculturales vinculados a la prostitución en la región, constituye un tema de sostenida presencia en el medio. Existen en el campo historiográfico, además, fundamentales coincidencias respecto al derrotero seguido por la trata de blancas y a la ambigüedad de un discurso oficial que oscilaba entre ver la inmigración como algo positivo para poblar las extensas regiones deshabitadas del país, hasta presentarla como una lacra social íntimamente vinculada a una «calidad» demográfica no deseada. Reforzaba esta última hipótesis la sonada actividad en Buenos Aires y su conurbano de dos organizaciones de proxenetas judíos, la Sociedad de Varsovia -Zwi Migdal, a finales de la década de 1920- y la Asquenasum, aun cuando parece probada la connivencia de sus rufianes con altos funcionarios políticos de la Argentina ${ }^{3}$.

Un aspecto menos explorado, en cambio, lo constituye la validación eugenésica de las políticas vinculadas al control de la prostitución delineadas en el contexto de significativa medicalización de la sexualidad que

2. Para excelentes pinturas de la cuestión en ambas orillas del Río de la Plata, además de la reconocida obra: Guy, Donna. El sexo peligroso. La prostitución legal en Buenos Aires 1875-1955. Buenos Aires: Sudamericana; 1994, pueden visitarse: Huertas, Rafael. Prostitución y espacio urbano: sobre la medicalización de la «mala vida» en Buenos Aires (1900-1920). Asclepio. 1990; 42 (2): 211-221. Carretero, Andrés. Prostitución en Buenos Aires. Buenos Aires: Corregidor; 1995. Grammático, Karin. Obreras, prostitutas y mal venéreo. Un Estado en busca de la profilaxis. In: Gil Lozano, Fernanda; Pita, Valeria Silvina; Ini, María Gabriela, dirs. Historia de las mujeres en la Argentina. Siglo XX. Buenos Aires: Taurus; 2000, vol. 2, p. 117-135. Goldar, Ernesto, La mala vida. In: Vázquez Rial, Horacio, dir. Buenos Aires 1880-1930. La capital de un imperio imaginario. Madrid: Alianza Editorial; 1996, p. 228-253. Gutman, Margarita y Reese, Thomas, eds. Buenos Aires, 1910. El imaginario para una gran capital. Buenos Aires: Eudeba. 1999. Múgica, María Luisa. Sexo bajo control. La prostitución reglamentada en Rosario entre 1900 y 1912. Rosario: Universidad Nacional de Rosario; 2001. Trochon, Ivette. Las mercenarias del amor. Prostitución y modernidad en el Uruguay (1880-1932). Montevideo: Taurus; 2003. Trochon, Ivette. Las rutas de Eros. Montevideo: Taurus; 2006. Las características físicas y localización de los prostíbulos en Buenos Aires, fueron tratadas en: Caride Bartrons, Horacio. Apuntes para una geografía de la prostitución en Buenos Aires 1904-1936. Seminario de Crítica 2009 [citado 17 Mar 2011]; 162: 2-48. Disponible en: http://www.iaa.fadu.uba.ar/publicaciones/ critica/0162.pdf.

3. Guy, n. 2, p. 154, entre otras. 
impregnó al país desde las primeras décadas del siglo XX. En este marco, y tal como veremos en las páginas siguientes, el estudio de la sexualidad, concebida como un par indisociable de la reproducción según la perspectiva biotipológica de oriente católico a la cual adscribió el eugenismo argentino, nos invita a ahondar en las intromisiones del poder público de diverso signo en la esfera de las sexualidades, autorizadas y prohibidas ${ }^{4}$. Así, al lado de la visualización del sexo como medio instrumental ineludible para lograr la generación de una descendencia sana y vigorosa, que asegurara la continuidad del «ser nacional» convivía una imagen del sexo como algo vergonzante, identificado con la sublevación, la insurrección y el desorden. Un monstruo bifronte a quien indistintamente se debía idolatrar, temer, civilizar, o quizás también, ignorar. Lugar donde se advierte aquella zona de sombra, de opacidad, de secreto, señalada por Phillipe Ariès ${ }^{5}$. Y fue precisamente en esta zona, y en las subjetividades en ella involucradas, donde el poder - en sus manifestaciones legales, judiciales, policiales, confesionales, en definitiva, represivas y metapartidarias - actuó con mayor eficacia.

Socavando sobre estas cuestiones desde el (bio)poder, la prostitución fue sistemáticamente asociada a la propagación de la sífilis, la blenorragia y el chancro blando, ocupando la primera enfermedad de esta tríada un lugar preferencial en el hipotético escenario de la degeneración de la raza. La urgencia de combatirla no era tanto entonces por la salud del cuerpo de la prostituta ni la de su descendencia (en general, ilegítima), sino por la de quienes, utilizando sus servicios, hicieran peligrar la salud de sus futuros hijos (legítimos) al transmitirles las consecuencias del morbo venéreo adquirido por el contacto sexual.

4. Para un análisis de la tensión entre sexualidades autorizadas y prohibidas en el contexto latino, ver: Miranda, Marisa. Controlar lo incontrolable. Una historia de la sexualidad en Argentina. Buenos Aires: Biblos; 2011. Las características de la línea eugénica adoptada por la ortodoxia argentina fueron tratadas en: Miranda, Marisa; Vallejo, Gustavo, comps. Darwinismo social y eugenesia en el mundo latino. Buenos Aires: Siglo XXI; 2005. Vallejo, Gustavo; Miranda, Marisa, comps. Políticas del cuerpo. Estrategias modernas de normalización del individuo y la sociedad. Buenos Aires: Siglo XXI; 2007. Vallejo, Gustavo; Miranda, Marisa. Derivas de Darwin. Cultura y política en clave biológica. Buenos Aires: Siglo XXI; 2010. Una referencia obligada en la materia son los textos: Stepan, Nancy Leys. The hour of eugenics. Ithaca-London: Cornell University Press; 1991. García González, Armando; Álvarez Peláez, Raquel. En busca de la raza perfecta, Madrid: CSIC; 1999. Scarzanella, Eugenia. Italiani malagente. Inmigrazione, criminalità, razzismo in Argentina, 1890-1940. Milano: Franco Angeli; 1999.

5. Ariès, Phillipe. Ensayos de la memoria 1943-1983. Bogotá: Grupo Editorial Norma; 1996 (en especial el capítulo «El secreto», p. 35-52). 
Para afrontar esa temida regresión disgenésica ${ }^{6}$, se implementó en diversos Estados - entre los cuales, cabe anticipar, la Argentina se convertiría en un faro latinoamericano- un programa de lucha antivenérea de tinte eugenésico, finalmente articulado en este país a través de tres vías fundamentales y complementarias de acción: la institucional específica; la institucional no específica y la legislativa.

La primera vía, la institucional específica (específica, claro está, en materia de divulgación de la eugenesia), quedaría inaugurada a partir de la consolidación en el medio local de la vertiente biotipológica enunciada en Italia por el endocrinólogo fascista Nicola Pende y corporizada en la Asociación Argentina de Biotipología, Eugenesia y Medicina Social, entidad fundada en la década del año 30 y que contara con Pende como Primer Miembro Honorario. Luego de 1945, la Sociedad Argentina de Eugenesia también se encargaría de reafirmar aquel posicionamiento, concentrándose en la articulación de mecanismos eficaces para lograr la detección anticipada de posibles perturbadores del orden social. Las mujeres que ejercían la prostitución no pudieron escapar fácilmente, claro está, de esa especie de peligrosidad predelictual que se les imputaba, aun cuando la práctica de ese «oficio», realizada de manera individual, sin el sostén de una organización criminal detrás, no era tipificada como delito.

La segunda vía, la no específica, precedió a la primera en el tiempo y allanó el camino para la instalación de la eugenesia como medio para lograr el descenso del morbo venéreo y, paralelamente, el control social de base biológica. La institución emblemática de esta vía fue la longeva Liga Argentina de Profilaxis Social, fundada en 1921 por el doctor Alfredo Fernández Verano y cuya incesante actividad se extendió en el tiempo hasta las postrimerías de la década de 1960 .

Finalmente, un tercer estadio fundamental en la profilaxis venérea tal cual fuera instaurada en el país, lo constituye el discurso médico-legal fortalecido desde estas instituciones y que le otorgara legitimidad científica al discurso político organizado en pos del combate de la prostitución que se pretendió garantizar con la ley abolicionista de Profilaxis de las Enfermedades Venéreas, sancionada en 1936 bajo el número $12.331^{7}$.

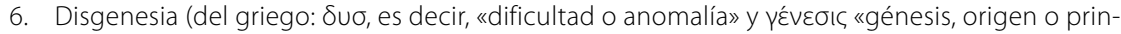
cipio de algo») resulta, entonces, un concepto opuesto a eugenesia.

7. Cabe recordar aquí que las posturas básicas en la materia pueden sintetizarse en tres regímenes: el «abolicionista», el «reglamentarista» y el «prohibicionista». El sistema «abolicionista» consiste
} 
El argumento utilizado para articular el control del comercio sexual bajo pretexto eugénico radicaba en que toda prostituta estaba enferma y que, infectando a un futuro padre de familia legalmente constituida, hacía peligrar la salud de una descendencia legítima a quien era necesario proteger para el bien de la Nación ${ }^{8}$. De esta manera, el «fin social» de la reproducción implicaba el deber social de garantizar «cualitativamente» sus frutos, en pos de un colectivo futuro e inasible, llamado «raza».

Un ascético discurso médico, basado en algunas certezas científicas y otras tantas aseveraciones dogmáticas, impugnaba así, a medias, el ejercicio de la prostitución. Y decimos ello, puesto que el sistema abolicionista tal como fue reglamentado en Argentina exponía en el tema una disfunción fundamental: el contagio venéreo no sólo se daba, claro está, a partir de una organización empresarial del comercio sexual; y, puesto que la prostituta «cuentapropista» tampoco quedaba exenta de contagiar a su cliente, el tema de la salud (de éste, de sus hijos o de ella misma) pasaba a un segundo plano. Retoma fuerza, entonces, la hipótesis que vincula a la biopolítica implementada en torno al tratamiento dado a la prostitución con cierta estrategia de legalización de jerarquías sociales.

Llegados a este punto, la tensión entre Eros y Tánatos quedaba convertida en una cuestión de Estado. El conflicto entre Eros, el dios responsable de la atracción sexual, del amor y del sexo en la mitología griega; y Tánatos, como personificación de la muerte no violenta, pretendió ser resuelto a partir de discursos y praxis vinculados a la normalización de la sexualidad. La defensa social contra el flagelo biológico avanzó más allá de una encomiable lucha contra la enfermedad en sí, hacia la estigmatización de los cuerpos «prostituidos» procurando afianzar, de ese modo, el rol de

en un régimen que sin llegar a la punición del simple ejercicio de la prostitución —como lo hace el sistema «prohibicionista»— no admite la reglamentación de los prostíbulos — como propone el sistema «reglamentarista»— sino que tiende a la supresión de los mismos.

8. Frecuentemente se reiteraba la consigna «toda prostituta es sifilítica, por el solo hecho de ejercer su profesión». Al respecto, ver, por ejemplo, Fernández Verano, Alfredo. Liga Argentina de Profilaxis Social. La Semana Médica. 1921; 28 (23): 683-687 (686). Estas ideas fueron retomadas durante los debates parlamentarios de la Ley de Profilaxis Antivenérea, en los cuales Enrique Mouchet afirmó: «Mejor es que se sepa esta verdad general: que toda mujer que ejerce la prostitución, sea en casa reglamentada o sin ninguna reglamentación, sin excepción, por ser prostituta, está enferma, fatalmente enferma y es contagiosa». Diario de Sesiones de la Cámara de Diputados Año 1936. Tomo IV. Buenos Aires: Imprenta del Congreso Nacional; 1937, p. 936. 
subalternidad que les fue otorgado por una elite visiblemente preocupada por sostener una estratificación social en crisis.

\section{Mal venéreo y defensa social}

Alfredo Fernández Verano, unido a otros reconocidos profesionales como José C. Belbey, Osvaldo Loudet, Julio Iribarne, Carlos S. Damel, Oreste Calcagno, José J. Puente, Páride T. Panza, Alberto Cildoz, Oscar Bonfiglio, Vicente A. Fiori, Julio Prebisch, Horacio C. Trejo, Pedro Pinto y Marcos A. Victoria daban forma, en 1921, a la Liga Argentina de Profilaxis Social. En la Junta Consultiva de esta institución también llegaron a actuar, entre otros, Gregorio Aráoz Alfaro, Mariano R. Castex, Alfredo L. Palacios, Augusto Bunge, Manuel V. Carbonel, Emilio R. Coni, Joaquín V. González, Estanislao Zeballos y José Ingenieros ${ }^{9}$. De estos nombres se advierte que, desde sus comienzos, la Liga Argentina de Profilaxis Social estuvo muy lejos de constituir un pensamiento heterodoxo; conformando, sin más, una institución altamente representativa del estado del campo científico local. Situación ésta que se mantuvo constante en el transcurso del tiempo, pese a los ulteriores conflictos entre Fernández Verano y la corporación médica ${ }^{10}$.

Ahora bien, la temprana adscripción de Fernández Verano a la eugenesia era notoria. Precisamente, ya en su tesis doctoral ${ }^{11}$, apadrinada por Emilio Coni, se ocupaba de ella en su primer capítulo, publicado años después en forma de libro ${ }^{12}$. Finalmente, la tesis íntegra - con ínfimas variaciones respecto al original- vio la luz en 1939, con prólogo del eugenista Nicolás Greco $^{13}$.

Por otra parte, los fines de la Liga ideada por Fernández Verano - fundada bajo el patrocinio del Círculo Médico Argentino y del Centro de Estudiantes

9. Liga Argentina de Profilaxis Social. Por la salud y el vigor de la raza. Plan de defensa social contra las enfermedades venéreas. Buenos Aires: Imprenta Mercatali; 1921, portada.

10. Fernández Verano, Alfredo. La Liga Argentina de Profilaxis Social y el Colegio de Médicos de la Capital Federal. La Semana Médica. 1933; 40 (19): 1628-1634.

11. Fernández Verano, Alfredo. La Medicina Social en la República Argentina. Buenos Aires: Universidad de Buenos Aires; 1921.

12. Fernández Verano, Alfredo. Las doctrinas eugénicas (ensayo de sistematización). Buenos Aires: edición del autor; 1929.

13. Fernández Verano, Alfredo. Para una patria grande un pueblo sano. Buenos Aires: Talleres Gráficos Compañía General Fabril Financiera; 1939. 
de Medicina - fueron anticipados en ocasión de la conferencia inaugural, donde básicamente se enfatizó sobre la relación entre enfermedad y defensa social. La disertación de marras se editó bajo el sugerente título «Por la Salud y el Vigor de la Raza. Plan de Defensa Social contra las Enfermedades Venéreas» ${ }^{14}$. En ese marco, Fernández Verano exclamó su admiración «a la naturaleza» que, mediante los abortos espontáneos que producía la sífilis, no hacía sino «subordinar un mal a otro mayor» ${ }^{15}$.

Desde su plan de acción, esta institución proclamó la necesidad de combatir las enfermedades venéreas en pos de la preservación y mejora de la raza a partir de la divulgación popular de diversas medidas profilácticas. En este sentido, facilitó la consolidación de un discurso hegemónico en ciernes, focalizado en la prostitución como un aspecto esencial en políticas de defensa social diseñadas en Argentina durante la primera mitad del siglo XX.

A partir de 1935, merced a la instauración del Día Antivenéreo en Argentina, la Liga comenzó a organizar sistemáticamente eventos de significativa repercusión, ampliando sus horizontes hasta insertarse firmemente en el marco internacional. Esta circunstancia le permitió articular sus premisas con las emanadas en el contexto de las redes eugénicas regionales que tuvieron a este país latinoamericano como centro de irradiación regional de las proclamas biopolíticas organizadas en la Europa latina.

Durante la primera celebración de esa índole, conjunta con Brasil y Uruguay, se convino establecer como Día Antivenéreo al primer domingo de septiembre de cada año ${ }^{16}$. A los discursos pronunciados por el entonces Director del Departamento de Higiene Sexual del Ministerio de Salud Pública de Montevideo, Héctor del Campo; del Vicepresidente de la Liga Brasileña de Higiene Mental, Julio Porto-Carrero y del Presidente de la Liga Argentina de Profilaxis Social, Fernández Verano, deben agregarse los del Profesor Titular de Dermatosifilografía de la Facultades de Ciencias Médicas de La Plata y Buenos Aires, Nicolás Greco; de la Presidenta de la Comisión de moralidad pública de la Alianza Internacional de Mujeres, Paulina Luisi; y de Alicia Moreau de Justo. Los aportes de las socialistas Luisi y Moreau de Justo se basaron en proponer la educación sexual como medida prioritaria

14. Fernández Verano, Alfredo. Conferencia inaugural. In: Liga Argentina, n. 9, folleto 1, p. 3-16.

15. Fernández Verano, n. 14, p. 7.

16. Liga Argentina de Profilaxis Social. El Día Antivenéreo. In: Por la salud de la raza. Discursos pronunciados en el gran acto público efectuado en el Teatro Colón, de Buenos Aires, en celebración del Día Antivenéreo, 7 de septiembre de 1935. Buenos Aires: s. e.; 1936, p. 3-5 (4). 
para combatir los males venéreos; mientras que la vertiente más dura sobre la cuestión quedaría expresada en la alocución de Nicolás Greco, para quien la sífilis generaba «haraganes, desleales, lenguaraces, avaros, caraduras», de manera tal que se auguraba no poder despoblar «las cárceles de delincuentes, ni los hospicios de dementes, ni los hospitales de enfermos, ni los colegios o la sociedad entera de anormales, ni los tribunales de pleitos, ni la iglesia de penitentes» ${ }^{17}$ si no se llevaba a cabo una intensiva profilaxis de la sífilis, como «principal proveedora de tanto elemento anormal» ${ }^{18}$.

Las repercusiones de esta campaña en el exterior permitieron que a la segunda celebración de este Día, el 7 de septiembre de 1936, se adhirieran siete países más, llegando en tan sólo un año a incrementarse en más del triple el número originario de los impulsores de la conmemoración. Esa ampliación incluyó a Bolivia, Chile, Costa Rica, Paraguay, Perú, Portugal y Venezuela ${ }^{19}$. Los Estados Unidos tomaron la propuesta, aunque se distanciaron de ella en fecha y nombre, utilizando el poco creíble argumento — «razones de orden climatológico»- esgrimido por su Director General del Departamento de Salud Pública, Thomas Parran ${ }^{20}$.

Ya por entonces, tanto para el Subsecretario de Beneficencia del Ministerio de Relaciones Exteriores y Culto argentino, Dívico Alberto Fürnkorn, como para el presidente de la Comisión de Higiene y Asistencia Social de la Cámara de Diputados de la Nación, Benito Soria, el problema venéreo era, sin más, una cuestión eugénica ${ }^{21}$. En ese orden de ideas, desde 1931 la Liga había instalado el primer consultorio prenupcial existente en Argentina, donde en 5 años fueron examinados 2.200 «aspirantes al matrimonio» que voluntariamente solicitaron sus servicios. La Estación Profiláctica organizada por aquélla en Capital Federal y que, en 3 años habría prestado sus servicios a más de 6.000 personas, sirvió a su vez de modelo para la

17. Greco, Nicolás. El Día Antivenéreo. In: Liga Argentina, n. 16, p. 23-27 (26).

18. Greco, n. 17.

19. Liga Argentina de Profilaxis Social. Significado del Día Antivenéreo. In: Hacia la extinción de un flagelo social. Discursos pronunciados en el gran acto público efectuado en el Teatro Colón, de Buenos Aires, en la segunda celebración del Día Antivenéreo septiembre 7 de 1936. Buenos Aires: Talleres Gráficos de la Compañía General Fabril Financiera; 1937, p. 3-5 (4).

20. Liga Argentina de Profilaxis Social. Apéndice. Mensajes de Adhesión. In: Liga Argentina, n. 19, p. 53.

21. Fürnkorn, Dívico Alberto. Palabras del Doctor Dívico Alberto Fürnkorn. In: Liga Argentina, n. 19, p. 7-11. Soria, Benito. Discurso pronunciado por el Doctor Benito Soria. In: Liga Argentina, n. 19, p. 13-17. 
instalación de una estación idéntica en Bahía Blanca ${ }^{22}$. Al respecto, cabe señalar un curioso dato que presenta lo exigente de las evaluaciones prenupciales hechas por la Liga: de esos aspirantes, tan sólo el 3\% era «apto» para el matrimonio ${ }^{23}$.

Ahora bien, en septiembre de 1937 tuvo lugar la tercera celebración del Día Antivenéreo, oportunidad en la cual el número de países adherentes se iría estabilizando ${ }^{24}$. Al año siguiente el Día Antivenéreo ya fue considerado una «fecha panamericana» ${ }^{25}$. La ampliación del impacto geográfico de la labor de la Liga Argentina llevó a titular la publicación de la quinta celebración del Día Antivenéreo con el omnipotente título de «Una cruzada continental en defensa de la salud de la raza» ${ }^{26}$. A esta celebración se incorporaría un reconocido penalista español en el exilio, Luis Jiménez de Asúa, quien instalado en Argentina, continuará durante años cercano al accionar de la Liga. La prostitución, una «degeneración del amor», era considerada por Jiménez de Asúa en directa asociación con la sífilis, uno de los tres grandes morbos que despoblaban a la humanidad. De ahí que la lucha de la Liga apuntaba, según él, a tres aspectos: el problema eugenésico, el problema del amor y el problema jurídico, es decir, de «cómo reglamentar la eugenesia y cómo reglamentar el matrimonio y la procreación» ${ }^{27}$.

En este marco, durante los fastos de 1940 realizados en conmemoración del sexto aniversario del Día Antivenéreo, se retomaría la consigna que imponía tender a la «eugenización del continente», como respuesta

22. Fernández Verano, Alfredo. La acción privada en la lucha antivenérea. In: Liga Argentina, n. 19, p. 37-41 (38).

23. Fernández Verano, Alfredo; Ascheri, Armando; Fairstein, David. Liga Argentina de Profilaxis Social. Consultorio prenupcial. Resultados obtenidos en los tres primeros años de funcionamiento. La Semana Médica. 1934; 41 (49): 1791-1794 (1794).

24. Liga Argentina de Profilaxis Social. Higiene sexual. Discursos pronunciados en el gran acto público efectuado en el Teatro Politeama, de Buenos Aires, con motivo de la tercera celebración del Día Antivenéreo septiembre 28 de 1937. Buenos Aires: Compañía Impresora Argentina; 1938.

25. Liga Argentina de Profilaxis Social. El Día Antivenéreo. Fecha panamericana. In: El Día Antivenéreo. Discursos pronunciados en el gran acto público efectuado en el Teatro Avenida, de Buenos Aires, con motivo de la cuarta celebración del Día Antivenéreo septiembre 5 de 1938. Buenos Aires: Compañía Impresora Argentina; 1939, p 3-5.

26. Liga Argentina de Profilaxis Social. Una cruzada continental en defensa de la salud de la raza. Discursos pronunciados con motivo de la quinta celebración anual del Día Antivenéreo septiembre 3 de 1939. Buenos Aires: Compañía Impresora Argentina; 1940.

27. Jiménez de Asúa, Luis. Conferencia del doctor Luis Jiménez de Asúa. In: Liga Argentina, n. 26, p. 11-14 (12-13). 
americana a la guerra que se estaba desarrollando en Europa, y que, se afirmaba operaba como evidente factor disgénico ${ }^{28}$. En esta empresa ya estaban involucrados Uruguay, mediante el Departamento de Higiene Sexual del Ministerio de Salud Pública; Brasil, a través de la Liga Brasileña de Higiene Mental de Río de Janeiro y de la Inspección de Profilaxis de la Sífilis y Enfermedades Venéreas del Estado de San Pablo; Perú, gracias a la acción de la Liga nacional de Higiene y Profilaxis Social; Chile, merced a la tarea de la Dirección del Departamento de Higiene Social de la Dirección General de Sanidad; Paraguay, por intermedio del Instituto de VenéreoSífilis de Asunción; Bolivia, mediante la Dirección de Sanidad Militar y el Departamento Nacional de Higiene y Salubridad Pública; Venezuela, a través del Ministerio de Sanidad y Asistencia Social y Costa Rica, merced a la gestión de Salubridad Pública y Protección Social. Fernández Verano insistía en la inclusión en este listado de los Estados Unidos de América, aún cuando no parecen haber sido suficientemente fluidos los vínculos entre su institución y el sistema sanitario norteamericano ${ }^{29}$.

Por su parte, las adhesiones al acto habidas en 1941 comprendieron las de la Oficina Sanitaria Panamericana (Washington), Bolivia, Costa Rica, Paraguay, Brasil y Uruguay ${ }^{30}$.

La décimo primera celebración del Día Antivenéreo, llevada a cabo en 1945 contaría además con la incorporación de México, corporizada a través de la disertación de su Secretario de Embajada ${ }^{31}$; mientras que, reafirmando la relación entablada entre sífilis, prostitución y eugenesia, la República de Bolivia participará oficialmente a través de un delegado del

28. Liga Argentina de Profilaxis Social. Hacia la eugenización del continente. In: América contra el peligro venéreo. Discursos pronunciados con motivo de la sexta celebración anual del Día Antivenéreo septiembre $1^{\circ}$ de 1940. Buenos Aires: Compañía Impresora Argentina; 1941, p. 3-4.

29. Fernández Verano, Alfredo. En la 60 celebración del Día Antivenéreo. In: Liga Argentina, n. 28, p. 5-8 (7-8). Liga Argentina de Profilaxis Social. Apéndice. Mensajes de Adhesión. In: Liga Argentina, n. 28, p. 27-32 (32).

30. Liga Argentina de Profilaxis Social. Apéndice. Mensaje de Adhesión. In: Por la salud de América. VII celebración anual del Día Antivenéreo septiembre 2 de 1941. Buenos Aires: Guillermo Kraft; 1942, p. 31-35.

31. Muñoz Zapata, José. Discurso de D. José Muñoz Zapata. Secretario de la Embajada de los Estados Unidos Mexicanos. In: Liga Argentina de Profilaxis Social. El Día de la Higiene Social. Buenos Aires: s. e.; 1948, p. 11-13. 
Ministerio de Higiene, Salubridad y Previsión Social y de la recientemente creada Sociedad Boliviana de Eugenesia ${ }^{32}$.

$\mathrm{Al}$ año siguiente, ya bajo la presidencia de Juan Domingo Perón, se declararía Día de la Higiene Social al primer domingo del mes de septiembre; siendo ello anunciado con énfasis por el Secretario de Salud Pública de la Nación, Ramón Carrillo, durante la décimo segunda celebración del Día Antivenéreo ${ }^{33}$. Visiblemente emocionado por ver considerada su obra, Fernández Verano reconocería:

«Ha sido menester — es de estricta justicia destacarlo-, que llegase a regir los destinos del país un gobierno inspirado en la defensa de los verdaderos intereses de la patria y de su mayor riqueza, la salud de sus habitantes, para que, después de un cuarto de siglo de estériles promesas, se adopte una política positiva en materia sanitaria» ${ }^{34}$.

La afinidad de Fernández Verano con el gobierno peronista quedaría recompensada a través de su designación como «delegado de la Secretaría de Salud Pública de la Nación» ante ese mismo evento ${ }^{35}$. En esa oportunidad, el vehemente Presidente de la Liga no dudó en denunciar un anterior «complot táctico» en contra de la profilaxis antivenérea, que hasta ese momento había estado «en manos de los enemigos más acérrimos de toda profilaxis», a quienes consideraba «más peligrosos que los mismos gérmenes morbosos» que se trataba combatir y responsabilizaba de un incremento del $700 \%$ de los casos de sífilis ${ }^{36}$.

Paralelamente, Carlos Bernaldo de Quirós, el fundador de la Sociedad Argentina de Eugenesia aunque disertante en esta ocasión en carácter de Delegado de la Sociedad Mexicana de Eugenesia propuso, sin más: «higienizar la mente de los niños, en su condición de artífices naturales de su propio destino, de conservadores de su capital humano, de futuros

32. Claros Escobar, Ángel. Discurso del Dr. Ángel Claros Escobar. Delegado del Ministerio de Higiene, Salubridad y Previsión Social de la República de Bolivia y de la Sociedad Boliviana de Eugenesia. In: Liga Argentina, n. 31, p. 15-19.

33. Carrillo, Ramón. Alocución pronunciada por el Excelentísimo señor Secretario de Salud Pública de la Nación, Dr. Ramón Carrillo. In: Liga Argentina, n. 31, p. 37-39.

34. Fernández Verano, Alfredo. En la XIla celebración anual del Día Antivenéreo. In: Liga Argentina, n. 31, p. $41-43$ (41-42).

35. Fernández Verano, Alfredo. Nuevas orientaciones de la lucha antivenérea. In: Liga Argentina, n. 31, p. 53-55.

36. Fernández Verano, n. 35, p. 55. 
esposos y padres responsables y de ciudadanos eugenésicos» ${ }^{37}$ y de las niñas, a quienes debía «regularse eugénicamente su higiene mental», para convertirlas en «artífices cósmicas de una gran nación», en su carácter de «futuras madres educadoras eugénicas», de «esposas hogareñas y directoras de reeducación social» ${ }^{38}$.

En 1947, durante los fastos conmemorativos del primer aniversario de la instauración del Día Nacional de la Higiene Social, la relación entre la Liga y el gobierno de Juan Perón continuaba en el idilio, adhiriendo personalmente a estos festejos la esposa del primer mandatario, María Eva Duarte ${ }^{39}$. De esta celebración también participará la Sociedad Argentina de Eugenesia, entidad manifiestamente opositora al ideario peronista. Sin la presencia de Quirós, acudió al evento Samuel Madrid Páez como delegado de esta Sociedad, circunstancia donde revitalizó una terrible proclama tan cara a Occidente: propender a la reproducción de los «individuos superiores» ${ }^{40}$. Cabe destacar así que pese al notorio descrédito en el que - con justiciahabía caído la eugenesia a nivel internacional luego del Holocausto, en nuestro país se insistía en anclar en ella las políticas públicas vinculadas a la erradicación de los males venéreos.

Por su parte, la Liga sostenía incólumes sus objetivos, enunciados desde su fundación, que iban desde «evitar y combatir la propagación de las enfermedades venéreas» hasta luchar contra la «pornografía, ya sea escrita, hablada o figurada», y se concentraban básicamente en la faz divulgadora de las medidas de prevención contra el morbo venéreo ${ }^{41}$. La confluencia de estos objetivos con los enunciados por las instituciones específicamente encargadas de la divulgación y praxis de la eugenesia era total. Ello, lejos de ser un hecho anecdótico o circunstancial, no sólo afianza la hipótesis de la potestad de validación de diversas praxis higienistas por la disciplina encargada de la «mejora de la raza», sino también la de su significativo grado de impregnación en la discursividad científico-política local, expuesta, en el caso en análisis, a partir de la lucha antivenérea.

37. Bernaldo de Quirós, Carlos. En la conmemoración del Día Antivenéreo. In: Liga Argentina, n. 31, p. 57-60 (58).

38. Bernaldo de Quirós, n. 37, p. 58-59.

39. Liga Argentina, n. 31, p. 93.

40. Madrid Páez, Samuel V. Alocución del Dr. Samuel V. In: Liga Argentina, n. 31, p. 87-90 (89).

41. Liga Argentina, n. 9. 


\section{La eugenesia y el anclaje biopolítico del abolicionismo}

La integración entre enfermedad venérea y disgenesia se convirtió en una constante del eugenismo argentino e incluyó, también, a líneas de pensamiento progresista cuyas inquietudes, bien diferenciadas en materia política de las propuestas ortodoxas, coincidieron sin embargo con éstas en ciertos aspectos vinculados al anclaje eugénico de la gestión de la salud pública.

Ahora bien, el cuello de botella que tempranamente se advierte en materia de tratamiento diferenciado del ejercicio de la prostitución individual respecto al de la prostitución organizada halla en parte su fundamento en el conflicto moral que para algunos suponía su prohibición lisa y llana. En efecto, a comienzos de la segunda década del siglo XX, el radical Leopoldo Bard retomaba las antiguas consignas que impugnaban la «castidad exagerada» vinculándola con la «perversión» del onanismo:

«¿De qué vale esa castidad exagerada, si luego la continencia en esas niñas, trae aparejada una masturbación, con pensamientos llenos de lascivia y lujuria? ¡Es tan difícil mantener la fuerza del alma y la del cuerpo! El himen estará intacto, no habrá sido profanado por el contacto sexual, pero ello será a costa de tocamientos, de orgasmos producidos por excitaciones continuas. La mujer, aunque virgen, puede estar pervertida, a causa de esta exagerada estimación que se hace de la virginidad, lo que hace que se desprecie a la que la haya perdido» ${ }^{42}$.

Entre ambos «males», y ante la conveniencia de evitar la práctica masturbatoria, Bard se inclinaba decididamente por la prostitución ${ }^{43}$. La educación sexual, un eje medular de la acción propuesta por Bard, debía iniciarse a partir de los 17 años. A los menores, de entre 13 y 15 años y sobre los cuales aseguraba que las enseñanzas aún no daban resultados, recomendaba el ejercicio esforzado en pos de «defenderlos contra ellos mismos, poniéndolos en guardia contra los placeres solitarios» ${ }^{44}$. Paralelamente, el sexólogo eugenista Lázaro Sirlin, reclamaba la necesidad de prohibir legalmente el azar en la reproducción conforme pautas de nor-

42. Bard, Leopoldo. Sobre castidad sexual. Comentarios. La Semana Médica. 1920; 27 (19): 622-625 (623).

43. Bard, n. 42, p. 624.

44. Bard, Leopoldo. Educación especial y profilaxis antivenérea en los centros obreros. La Semana Médica. 1920; 27 (17): 562-565 (564). 
malidad que vendrían a sustentar «conveniencias raciales», debiéndosele inculcar a los adolescentes la «importancia racial de su unión», criterio de acuerdo al cual deberían realizar su elección de pareja ${ }^{45}$.

Fernández Verano compartía básicamente la idea de que la procreación no era un derecho individual sino un deber social irrenunciable para los representantes de las «razas y clases superiores» que debía ser desalentado en las «inferiores» ${ }^{46}$. Sobre este sustrato, la lucha contra la prostitución como factor disgénico entablada desde la Liga, impulsaba desde la revisión de las prostitutas y el apoyo a la «mujer caída ${ }^{47}$, hasta la intervención pública abocada a la resolución de la «crisis de erotismo» que se reflejaba en la «libertad de costumbres modernas», tal como quedaba expuesta en «revistas y libros que hace algunos años no se hubiera atrevido a exponer el librero más audaz» ${ }^{48}$. Mediante la represión severa de la pornografía, unida a la propuesta de «penar la incultura verbal» en la calle, parajes públicos y tranvías, se intentaba desviar a los jóvenes de las «tentaciones de las prostitutas», y reforzar, a la vez, la idea de familia y el fomento a los matrimonios tempranos ${ }^{49}$.

En este contexto, y luego de diversas idas y venidas en torno a la oportunidad y conveniencia de reglamentar la prostitución, el socialismo presentaba al Congreso Nacional en 1933 un proyecto de Ley de Profilaxis Antivenérea elaborado por el diputado Ángel Giménez, preguntándose retóricamente su autor:

«¿Dónde está el foco de este terrible mal que ataca y mutila al individuo, que destruye la familia, bastardea la raza y la sociedad, recargando con ingentes sumas los presupuestos de la asistencia?» ${ }^{50}$,

45. Sirlin, Lázaro. Algo sobre eugenia y educación. La Semana Médica. 1920; 27 (52): 875-877 (875).

46. Fernández Verano, n. 13, p. 46-47 y 58-59.

47. En este sentido, un proyecto de la Liga Argentina de Profilaxis Social previó la creación de la Asociación de Señoras para Rehabilitación y Regeneración de la «mujer caída». Fernández Verano, n. 13, p. 187.

48. Fernández Verano, n. 8, p. 685.

49. Sobre la edad «exacta» para casarse, Fernández Verano sostuvo la efectividad de una particular ecuación: la esposa debía tener la mitad del número de años del marido, más siete. Fernández Verano, n. 12, p. 24.

50. Giménez, Ángel. Profilaxis de las enfermedades venéreas. Proyecto de ley. La Semana Médica. 1933; 40 (45): 1459-1467 (1462). 
a lo que inmediatamente respondía: «Está en primer término en la prostitución, sin dejar de reconocerse otros factores de contaminación» ${ }^{51}$. Giménez aportaba su proyecto con el afán de merecer la consideración del «mundo civilizado» y para que por su intermedio, la fusión de los componentes del crisol de razas que era Argentina se hiciera «libres de taras adquiridas o hereditarias, y sin la marea que bastardea la especie, las enfermedades venéreas, el alcoholismo y otras lacras sociales». Es decir, finalmente, se hiciera «obra de eugenesia» ${ }^{52}$.

En la misma sintonía, Carlos Bernaldo de Quirós propuso criminalizar la prostitución. Para evitar el ejercicio del comercio sexual, y defender con ello al ciudadano de la «degeneración de la raza», íntimamente vinculada a la prostitución, a la tuberculosis, al alcoholismo y a la miseria, debía encararse la «profilaxis moral» desde la escuela ${ }^{53}$. Esa lucha antivenérea no sólo establecería obligaciones eugénicas y responsabilidades legales traducibles en resarcimientos económicos contra todos los propagadores del venéreo, sino también crearía el impedimento de salud, es decir, la exigencia obligatoria del certificado médico prenupcial, para impedir toda unión matrimonial entre seres «tarados» ${ }^{54}$.

En este sentido, también para la Liga Argentina de Profilaxis Social el Estado debía ocuparse con premura de obtener generaciones sanas y fuertes desde el nacimiento:

«QQué puede esperarse, para el progreso de una nación, de una multitud de idiotas, imbéciles, retardados mentales, deformes, inválidos, epilépticos, sordomudos, ciegos, criminales, etc., etc.?» ${ }^{55}$.

Desde ahí se propuso que así como se sometía a los jóvenes a un examen médico para cumplir con el servicio militar era menester $-\mathrm{y}$ con mayor razón- seleccionarlos antes de realizar la función reproductiva.

51. Giménez, n. 50.

52. Giménez, n. 50, p. 1467.

53. Bernaldo de Quirós, Carlos. Delincuencia venérea (Estudio eugénico-jurídico). Buenos Aires: edición del autor; 1934, p. 52-53.

54. Bernaldo de Quirós, Carlos. El delito del contagio venéreo. Anales de Biotipología, Eugenesia y Medicina Social. 1934; 2 (21): 10, 15-16. Ver, también: Beltrán, Juan Ramón. El delito de contagio venéreo en la legislación argentina. Anales de Biotipología, Eugenesia y Medicina Social. 1934; 2 (22): 9.

55. Fernández Verano; Ascheri; Fairstein, n. 23, p. 1792. 
La mentalidad eugénica reclamada debía estar acompañada de un claro sentimiento de «responsabilidad racial» 56 .

Obtenida en 1935 la media sanción de Diputados del proyecto de Giménez, Bernaldo de Quirós recomendó al Senado su despacho favorable, ya que era «una de las leyes de higiene y asistencia social más trascendentales con que contaría el patrimonio legislativo y sanitario de la República ${ }^{57}$. Si bien para este jurista el abolicionismo no era un remedio definitivo contra la prostitución como agente causal de la degeneración de la raza, sí era eficaz al momento de perseguir la morbilidad venérea, emancipar y dignificar a la mujer, estimular el sentido de la responsabilidad moral y física en el hombre y moralizar el ambiente social ${ }^{58}$. Quirós compartía con la mayoría de sus contemporáneos un lugar común: la visión de la prostitución como causa determinante de lesiones orgánicas de importancia, vinculadas directamente al proceso hereditario, ya que los «hijos de sifilíticos, de alcoholistas, de débiles mentales, y otras taras hereditarias» mantenían un constante interrogante sobre el «porvenir de la raza blanca en los destinos futuros de la Humanidad» 59 .

Obviando aquí las particularidades de sus debates parlamentarios, ya tratados en otro trabajo ${ }^{60}$, la Ley Antivenérea fue finalmente sancionada a fines de 1936 llevando el número 12.331 y se basó, en lo fundamental, en el mencionado proyecto de Ángel Giménez - quien cabe aclarar, también fue Secretario de la Sección Eugenesia de la Asociación Argentina de Biotipología, Eugenesia y Medicina Social ${ }^{61}-$, y en el de profilaxis venérea y obligatoriedad del certificado de salud prenupcial elaborado por Tiburcio Padilla. Este último también sostuvo su discurso desde el lugar común que constituía la eugenesia, y apelando al español Gregorio Marañón, enfatizó:

«si se selecciona a los sementales de las cabañas, si se escoge a los hombres más sanos y fuertes para dedicarlos al servicio militar o a realizar la guerra,

\footnotetext{
56. Fernández Verano; Ascheri; Fairstein, n. 23, p. 1792.

57. Bernaldo de Quirós, Carlos. A un paso del abolicionismo nacional. Anales de Biotipología, Eugenesia y Medicina Social. 1936; 3 (62): 3-4 (3).

58. Bernaldo de Quirós, n. 57.

59. Bernaldo de Quirós, Carlos. El determinismo económico en la fenomenología eugénico-social. Anales de Biotipología, Eugenesia y Medicina Social. 1936; 3 (67): 3-8, 20 (8)

60. Para profundizar, remitimos a: Miranda, Marisa. Prostitución y homosexualidad en Argentina: el discurso eugénico como sustrato teórico de biopolíticas represivas. In: Miranda, Vallejo, n. 4, p. 451-494.

61. Anales de Biotipología, Eugenesia y Medicina Social. 1933; 1 (1): 1.
} 
con cuánta mayor razón conviene tomar algunas medidas con los que serán futuros padres de familia» ${ }^{62}$.

Así las cosas, el abolicionismo de la ley prometía ventajas de orden «higiénico y moral», fundamentalmente teniendo en cuenta las particulares características de «nuestras» pobres prostitutas, bien diferenciadas, según Padilla, de una geisha y de una cocotte . $^{63}$.

Por otra parte, a mediados de agosto de 1936 el gobernador de la Provincia de Buenos Aires, Manuel Fresco, un ferviente admirador de Benito Mussolini, dictó un decreto mediante el cual dispuso la clausura temporaria de algunos prostíbulos situados en el conurbano bonaerense ${ }^{64}$; clausura que se levantaría luego de que estos establecimientos se adecuaran a la reglamentación dictada a fines de ese mes a partir de un estudio realizado por la Dirección General de Higiene ${ }^{65}$. Para Fresco, que no era abolicionista, resultaba indispensable mantener la prostitución reglamentada hasta tanto el pueblo aprendiera a defenderse de las enfermedades cuyos graves efectos para el individuo y para la especie se trataba de neutralizar.

En definitiva, la Ley de Profilaxis de las Enfermedades Venéreas preveía mecanismos de tinte coercitivo, como lo eran la presentación de certificado prenupcial obligatorio para los futuros cónyuges de sexo masculino, el tratamiento obligatorio de los enfermos y la hospitalización forzosa de quienes pudieran constituir un «peligro social»; y mecanismos preventivos, como la educación sexual. Sin embargo, curiosamente, el ejercicio de la prostitución individual no estaba punido ni reglamentado.

No obstante, y pese a la hermenéutica amplia con que se aplicó esa norma, las elites argentinas advirtieron su insuficiencia como dispositivo de control y «orden» de la sexualidad ${ }^{66}$; insuficiencia que además, puso en evidencia la tensión existente entre el modelo conservador de sociedad

62. Palabras de Tiburcio Padilla en Diario de Sesiones de la Cámara de Diputados Año 1935. Tomo IV. Buenos Aires: Imprenta del Congreso Nacional; 1936, p. 567-568.

63. Diario de Sesiones, n. 8, p. 932-933.

64. Boletín Oficial de la Provincia de Buenos Aires. 1936; 14 de Agosto: 13.854-13.856.

65. Boletín Oficial de la Provincia de Buenos Aires. 1936; 26 de Agosto: 14.349-14.350.

66. La ineficacia de la ley respecto a aspectos vinculados a su inobservancia por miembros de la corporación médica fue tempranamente puesta de manifiesto desde cierto sector de la izquierda argentina. Zamora, Antonio. Nota editorial. La irresponsabilidad médica. Cultura sexual y física. 1938; 9 (1): 513-514. 
tenido en mira al dictarla y el andamiaje constitucional de corte liberal que organizaba al país.

Por otra parte, esta ley cuenta en su haber con la curiosa imputación de responsabilidad sobre el eventual incremento de la homosexualidad masculina en las Fuerzas Armadas. El «escándalo de los cadetes» de 1942 puso de manifiesto una inaceptable vulnerabilidad sexual en sus miembros e hizo más visible aún la homofobia presente en ciertas instituciones de la sociedad argentina. Desde esta perspectiva se vio al episodio protagonizado por los cadetes del Colegio Militar de la Nación - quienes fueron descubiertos en reuniones gay - como una prueba cabal de la ineficacia del sistema abolicionista instaurado en 1936. En este sentido, retomando la tesis que veía a la prostitución como un antídoto para la homosexualidad, se responsabilizó del aparente cambio de orientación sexual de algunos miembros de las Fuerzas Armadas a la inexistencia de prostitución reglamentada ${ }^{67}$.

Ante esa situación, el gobierno de facto de Edelmiro J. Farell modificó la norma antivenérea en abril de 1944, mediante el Decreto 10.638. La prohibición del establecimiento de casas donde se ejerciera la prostitución - o casas de tolerancia - que disponía la Ley 12.331, quedó menguada mediante la posibilidad de su instalación, siempre y cuando se contase con autorización de la Dirección Nacional de Salud Pública y Asistencia Social y aprobación del Ministerio del Interior. Por su parte, la discusión originada ya desde 1937 respecto a si el ejercicio de la prostitución en forma individual estaba comprendido en la prohibición legislativa, quedó también resuelta por el Decreto de 1944 que dispuso, con más claridad que aquella norma, que no sería considerado delito, mientras no afectase el pudor público.

Este decreto, de corte reglamentarista, claramente tendía a contener a los soldados apostados en bases remotas, evitando el riesgo de que ante la abstención sexual con mujeres pretendieran saciar su apetito con hombres; y permitía, a su vez, el control médico de los burdeles. Admitiéndose el funcionamiento de prostíbulos, es decir, reglamentando la prostitución, se pretendía impedir que los buscadores de placer cayeran en «perversiones» como el onanismo y la homosexualidad. Los burdeles, claro está, en la medida en que estimulaban la heterosexualidad tradicional reforzaban a las instituciones del matrimonio y de la familia.

67. Para ahondar sobre este incidente, ver: Miranda, n. 60, p. 472-481. 
A poco de asumir el cargo de Presidente de la Nación Juan Domingo Perón, Ramón Carrillo, su Secretario de Salud Pública, entendió que la Dirección de Higiene Social debía ocuparse de la lucha antivenérea, en cuyo marco llevaría el Registro Nacional de Higiene Sexual ${ }^{68}$. Asimismo, cabe recordar que el Decreto 9863/46 apoyaba decididamente las disposiciones abolicionistas de la Ley de Profilaxis Social.

No obstante, en el ocaso de este gobierno, el Decreto 22.532/54 autorizó el funcionamiento de casas de tolerancia. Y, a la vez, tanto la Municipalidad como la Provincia de Buenos Aires hacían lo propio en materia de reglamentación de la prostitución; obligando a la inscripción de la pupila en un registro especial y a la instalación de un consultorio médico en cada prostíbulo $^{69}$. Meses más tarde, el régimen de facto que depuso a Perón dictó el Decreto 4863/55, retomando la postura abolicionista de la Ley de 1936.

\section{Reflexiones finales}

En esta pintura del tratamiento biopolítico de la prostitución en Argentina durante las primeras décadas del siglo XX se advierte tempranamente la insuficiencia de explicaciones monocausales. Así, sin pretender quitar relevancia a la evidente urgencia de entonces en combatir la trata de blancas generada por el accionar de diversas organizaciones de proxenetas instaladas en Buenos Aires, cabe también dar visibilidad a otros aspectos de una compleja red de intereses y mediaciones igualmente presentes.

En efecto, afianzada la medicalización de la sexualidad y ante la necesidad de afirmar los tradicionales roles de género que se temía perturbados a partir del ingreso de ideologías exógenas traídas por el aluvión inmigratorio, el abordaje de la prostitución se desmadró rápidamente de una cuestión meramente higiénica para transformarse en una cuestión eugénica. De esta manera, disparado desde un relato fundado en un hipotético bien común orientado a la constitución mítica de una «raza» sana y fuerte, se consolidó

68. Resolución 2708/1947. Funciones, deberes y atribuciones de las Direcciones de la Secretaría de Salud Pública de la Nación. Archivos de la Secretaría de Salud Pública de la Nación. 1947; 2 (11): 70-86 (82).

69. Decretos $2492 / 55$ de la Municipalidad de Buenos Aires y 4633/55 de la Provincia de Buenos Aires. 
en el país, bajo el nombre de eugenesia, una ideología que preconizaba la dicotomía de los cuerpos.

Resultan evidentes las fisuras entre los mandatos profilácticos y su corporización legislativa, que tan solo desarticuló la organización empresarial del comercio sexual y tuvo, en cambio, relativa incidencia en materia sanitaria; circunstancia que denota la subsistencia (¿intencionada?) de una relación jerárquica entre «cuerpos serviles» $\mathrm{y}$ «cuerpos servidos». En este contexto, la contienda entre Eros y Tánatos fue mediada (y resuelta a medias) por un discurso eugenésico persistente en el tiempo, más involucrado en el sostenimiento de roles y gradaciones sociales que en la instrumentación de políticas tendientes a mejorar la calidad de vida de los sectores más vulnerables, debilitándose así los anhelos originarios del progresismo argentino, de activa participación en la génesis de estos debates. 
Buenos Aires, between Eros and Thanatos. Prostitution as dysgenic threat (1930-1955)

Marisa A. Miranda . ...

1.--Introduction: female prostitution as dysgenic threat. 2.-Venereal illness and social defence. 3.-Eugenics and the biopolitical foundation of abolitionism. 4.-Final reflections.

ABSTRACT: This paper explores the medical-legal discourse around female prostitution that was articulated in Buenos Aires during the first decades of the 
20th century. It focuses on the use of different ideological resources and on the policies and laws that were introduced to gain control over the sex trade and describes parallelisms between prostitution, disease and crime. In fact, the capital of Argentina was internationally known as a centre of prostitution and white slave trafficking, and Jewish participation in these activities allowed the consolidation of xenophobic theories. Medicine and law, among other disciplines, made important inputs into a simplified approach to this problem. 